\title{
SI DELTA DOPED GaN GROWN BY LOW-PRESSURE METALORGANIC CHEMICAL VAPOR DEPOSITION
}

\author{
Jong-Hee Kim, Gye Mo Yang, Sung Chul Choi, Ji Youn Choi, \\ Hyun Kyung Cho, Kee Young Lim, Hyung Jae Lee \\ Semiconductor Physics Research Center and \\ Department of Semiconductor Science and Technology, \\ Chonbuk National University, Chonju 561-756, Korea
}

\section{Cite this article as: MRS Internet J. Nitride Semicon. Res. 4S1, G3.49(1999)}

\begin{abstract}
Si delta-doping in the GaN layer has been successfully demonstrated by low-pressure metalorganic chemical vapor deposition at a growth temperature of 1040 . Si delta-doping concentration increases and then decreases with an increase in delta-doping time. This indicates that delta-doping concentration is limited by the desorption process owing to much higher thermal decomposition efficiency of silane at high growth temperatures of GaN. In addition, it was observed that the use of a post-purge step in the ammonia ambient reduces Si delta-doping concentration. From capacitance-voltage measurement, a sharp carrier concentration profile with a full-width at half maximum of $4.1 \mathrm{~nm}$ has been achieved with a high peak concentration of $9.810^{18} \mathrm{~cm}^{-3}$.
\end{abstract}

\section{INTRODUCTION}

In the past decade, though many inherent problems originating from the heteroepitaxial growth in wide-bandgap III-V nitride materials exist, there have been considerable developments that have led to the remarkable demonstrations of high-brightness blue light emitting diodes [1,2], laser diodes [3], and high-frequency field-effect transistors (FETs) [4,5]. In particular, high-frequency FETs using GaN/AlGaN heterostructures have been realized using uniformly $\mathrm{Si}$ doped layers. In III-V compound semiconductors such as GaAs, FETs with characteristics superior to those of conventionally doped transistors have been fabricated using the delta-doping technique [6], which can achieve very narrow doping distribution along the epitaxial growth direction and up to now has been studied in a variety of materials and devices [7-9]. It was recently demonstrated that the inclusion of delta doping could improve laser stability in the face of temperature variations, in comparison with conventional quantum well lasers [10].

Even though the growth temperature of $\mathrm{GaN}$ in metalorganic chemical vapor deposition (MOCVD) is much higher than that of conventional III-V compound semiconductors, it is considered to be important to investigate delta-doping in III-V nitride materials for highperformance device applications. To our knowledge, up to now there are no reports about the application of delta-doping on III-V nitrides. In this letter, we demonstrate Si delta doping in 
GaN grown by low-pressure MOCVD and investigate the effects of delta-doping time and postdelta-doping purge step on carrier concentration.

\section{EXPERIMENTAL}

The samples used in this study were grown in a vertical rotating MOCVD reactor operating at 200 Torr. Trimethylgallium (TMG), ammonia $\left(\mathrm{NH}_{3}\right)$, and $100 \mathrm{ppm}$ silane $\left(\mathrm{SiH}_{4}\right)$ in hydrogen were used as $\mathrm{Ga}, \mathrm{N}$, and $\mathrm{Si}$ delta-dopant precursors, respectively. Substrates used in this experiment were c-plane sapphires, which were degreased in an organic solvent and then slightly etched in a hot solution of $3 \mathrm{H}_{2} \mathrm{SO}_{4}: 1 \mathrm{H}_{3} \mathrm{PO}_{4}$ for $10 \mathrm{~min}$. After the thermal cleaning in $\mathrm{H}_{2}$ environment at 1070 for $10 \mathrm{~min}$, a 20-nm-thick $\mathrm{GaN}$ buffer layer was deposited at 520 . Finally, the substrate temperature was raised to 1040 to grow the GaN overlayer. The V/IIf ratio for the GaN overlayer was 1560. Under these conditions, background doping was 810

n-type. The growth procedure for Si delta-doped $\mathrm{GaN}$ was as follows. First, an undoped $\mathrm{GaN}$ layer (1.7 ) was grown, after which TMG supply was stopped for $10 \mathrm{~s}$ on top of the GaN layer, while at that time ammonia was kept flowing. At this pre-delta-doping purge step, the surface became N-rich. This was followed by Si deposition for several tens of seconds under a Ga-free condition. The $\mathrm{SiH}_{4}$ flow rate was $24 \mathrm{nmol} / \mathrm{min}$. Then, we deposited an undoped GaN cap layer by two different methods: (1) post-delta-doping purge step for $10 \mathrm{~s}$ with flowing ammonia followed by the growth of a cap layer; and (2) direct growth of a cap layer without the post-deltadoping purge step. After the structure growth, a Schottky barrier was fabricated by thermal evaporation of gold through a shadow mask containing 620 dots. Aluminum was deposited

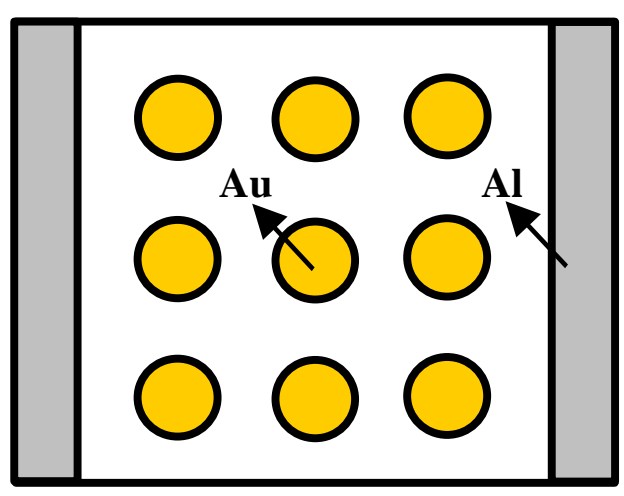

Fig. 1. Schematic diagram of fabricated devices for Si delta-doped GaN.

over a large area on the perimeter of the crystal face to serve as an ohmic contact as shown in Fig. 1. The carrier concentration was obtained by capacitance-voltage $(\mathrm{C}-\mathrm{V})$ measurement, which was performed with a $1 \mathrm{MHz}$ capacitance meter (Boonton 72-4B) at room temperature.

\section{RESULTS AND DISCUSSION}


The $\mathrm{C}-\mathrm{V}$ profiles of Si delta-doped GaN layers depending on the delta-doping time are shown in Fig. 2. Silane flow rate was $4 \mathrm{nmol} / \mathrm{min}$, and the pre- and post-purge times on both sides of delta doping were the same, i.e., 10 s. Figure 2 demonstrates that $\mathrm{Si}$ delta-doped GaN can be successfully grown using MOCVD. The maximum C-V concentration of Si delta-doped $\mathrm{GaN}$ does not increase with the increase in delta-doping time, in contrast to a strong linear dependence for Si delta-doped GaAs [11]. This shows that the Si delta-doping process might be dominated by the desorption of Si dopant from the nongrowing surface. The growth temperature of GaAs is lower than that of GaN. Therefore, as a result of low thermal decomposition efficiency of Si doping precursors, Si delta-doping concentration in GaAs is mainly determined by the adsorption process [11]. However, due to the high growth temperature of $\mathrm{GaN}$, the thermal
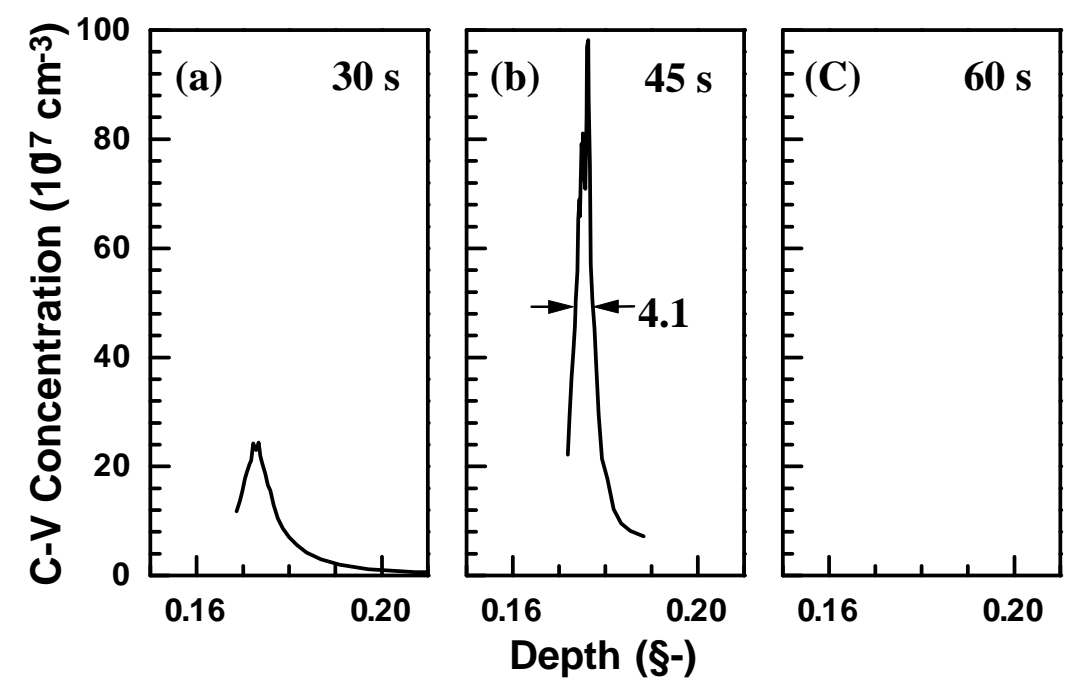

Fig. 2. C-V profiles of Si delta-doped GaN grown under different delta-doping times of (a) $30 \mathrm{~s}$, (b) $45 \mathrm{~s}$, and (c) $60 \mathrm{~s}$.

decomposition efficiency of Si doping precursors will be much higher than that in the case of GaAs. This means that the adsorption/desorption process can quickly approach equilibrium. $\mathrm{Si}$ delta-doping concentration is therefore determined by the equilibrium between the adsorption and desorption processes with the desorption step limiting. This eventually leads to the abovementioned relationship between the electron density and the delta-doping time.

On the other hand, silicon dopants ideally should be confined to a single atomic monolayer by the growth interruption technique. However, the doping profile will be broadened by dopant diffusion and segregation processes. Usually, the broadening of the doping profile is estimated from the full width at half-maximum (FWHM) of the C-V profile. In the case of GaN, the diffusion process may significantly affect the broadening of the doping profile because the growth temperature of $\mathrm{GaN}$ is much higher than that of conventional III-V compound semiconductors. As can be seen in Fig. 2(b), however, the FWHM of the C-V profile in Si deltadoped $\mathrm{GaN}$ is as narrow as $4.1 \mathrm{~nm}$. This sharp distribution profile is comparable with the values 
for Si delta-doped GaAs [12]. It indicates that the diffusion effect is small for Si delta doping in GaN by low-pressure MOCVD. Using optimized growth condition, the delta-doping technique in $\mathrm{GaN}$ has the feasibility of the fabrication of high performance electronic and opto-electronic devices.

The basic delta-doping procedure includes pre-purge, delta-doping, and post-purge steps. The function of pre-purge step is to completely suspend growth of the host material for minimized memory effect of growing species on subsequent delta-doping. The post-purge step minimizes the dopant memory effect, after which growth of the host material is resumed. However, in the case of delta-doped GaAs the use of the pre-purge and post-purge step in the group $\mathrm{V}$ containing ambient reduces $\mathrm{Si}$ delta-doping concentration [13]. Figure 3 shows the room-temperature $\mathrm{C}-\mathrm{V}$ profiles of $\mathrm{Si}$ delta-doped $\mathrm{GaN}$ layers grown under different post-deltadoping conditions. The pre-purge time before delta doping was $10 \mathrm{~s}$. During the delta-doping step on the nongrowing GaN surface, silane flow rate was $2 \mathrm{nmol} / \mathrm{min}$ and delta doping time was $60 \mathrm{~s}$. The $\mathrm{C}-\mathrm{V}$ profile corresponding to the $\mathrm{Si}$ delta-doped structure with a post-purge time of $10 \mathrm{~s}$ is shown in Fig. 3(a). It shows a FWHM of $11.6 \mathrm{~nm}$ and a maximum C-V concentration of 2.8

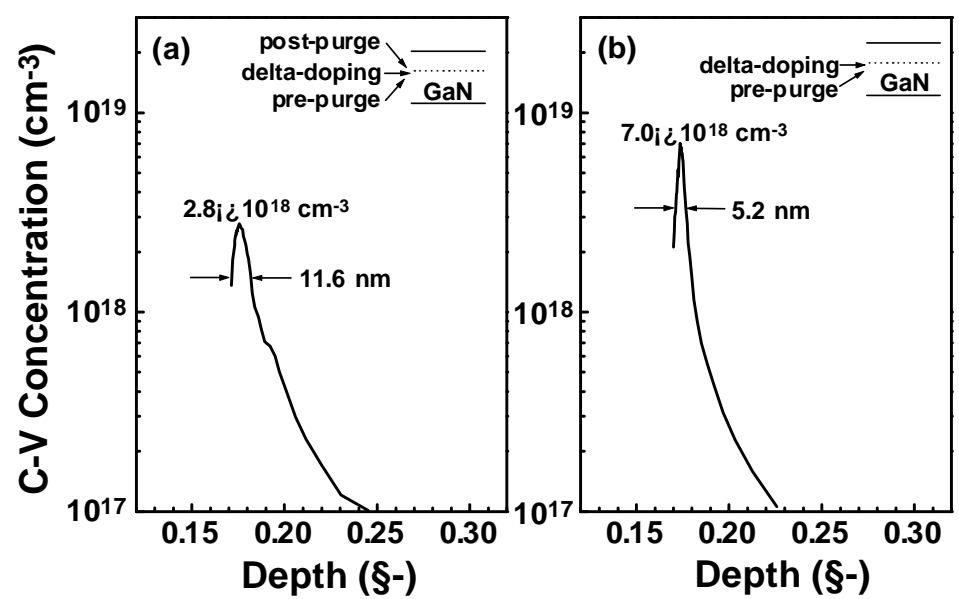

Fig. 3. Effect of post-delta-doping purge step on room-temperature C-V profiles of Si delta-doped GaN. (a) Ammonia post-purge (10 s) step after delta-doping. (b) Direct growth of GaN host material without post-purge step.

$10^{18} \mathrm{~cm}^{-3}$. On the other hand, Si delta-doped $\mathrm{GaN}$ without the post-delta-doping purge step shown in Fig. 3(b) has a much higher peak concentration of $710^{18} \mathrm{~cm}^{-3}$ than the structure obtained with the post-purge step. In addition, it indicates a sharp profile with a FWHM of 5.2 $\mathrm{nm}$. Obviously, when a post-purge step is included in the delta-doping sequence of GaN, Si evaporation will make it extremely difficult to achieve a higher Si delta-doping concentration. This phenomenon is similar to the reported result for Si delta-doped GaAs grown by MOCVD [13]. The above results confirm that the optimized delta-doping time and the delta-doping sequence without the post-purge step should be considered in order to maximize the delta-doping concentration. 


\section{CONCLUSION}

Si delta-doped GaN layers have been successfully grown by low-pressure MOCVD. From $\mathrm{C}-\mathrm{V}$ measurement, we found that the delta-doping of $\mathrm{Si}$ in $\mathrm{GaN}$ has a dependence on deltadoping time that differs from that of Si delta-doping in GaAs and that it may be dominated by the desorption process of Si. Additionally, to obtain a higher Si delta-doping concentration, it is essential to utilize the delta-doping sequence that does not have a post-delta-doping purge step.

\section{ACKNOWLEDGMENTS}

This work was partially supported by MSTK and by KOSEF through the Semiconductor Physics Research Center at Chonbuk National University, and by the Ministry of Education of Korea.

\section{REFERENCES}

1. P. Kozodoy, A. Abare, R. K. Sink, M. Mack, S. Keller, S. P. DenBaars, U. K. Mishra and D. Steigerwald: Mat. Res. Soc. Symp. Proc. 468, 481 (1997).

2. S. Nakamura, M. Senoh, N. Isawa, S. Nagahama, T. Yamada and T. Mukai: Jpn. J. Appl. Phys. 34, L1332 (1995).

3. S. Nakamura, M. Senoh, S. Nagahama, N. Isawa, T. Yamada, T. Matsushita, H. Kiyoku, Y. Sugimoto, T. Kozaki, H. Umemoto, M. Sano and K. Chocho: Jpn. J. Appl. Phys. 37, L309 (1998).

4. J. Burm, W. J. Schaff, L. F. Eastman, H. Amano and I. Akasaki: Appl. Phys. Lett. 68, 2849 (1996).

5. Q. Chen, M. A. Khan, J. W. Yang, C. J. Sun, M. S. Shur and H. Park: J. Appl. Phys. 69, 794 (1996).

6. S. Nakajima, K. Otobe and N. Shiga: IEEE Trans. Electron. Devices 39, 771 (1992).

7. E. F. Schubert: J. Vac. Sci. Technol. A 8, 2980 (1990).

8. G. Li and C. Jagadish: Solid State Electron. 41 (1997) 1207.

9. J.-H. Kim, D. H. Lim, G. M. Yang, Y. G. Shin, K. Y. Lim and H. J. Lee: Appl. Phys. Lett. 69, 1870 (1996).

10. O. Buchinsky, M. Blumin and D. Fekete: Appl. Phys. Lett. 72, 1484 (1998).

11. H. M. Shieh, W. C. Hsu and C. L. Wu: Appl. Phys. Lett. 63, 509 (1993).

12. E. F. Schubert, J. B. Stark, B. Ullrich and J. E. Cunningham: Appl. Phys. Lett. 52, 1508 (1988).

13. G. Li and C. Jagadish: Mater. Sci. Eng. B 33, 182 (1995). 